\title{
Human Gyrovirus-Apoptin Interferes with the Cell Cycle and Induces G2/M Arrest Prior to Apoptosis
}

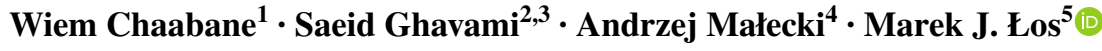

Received: 7 October 2016 / Accepted: 20 February 2017 / Published online: 6 April 2017

(C) L. Hirszfeld Institute of Immunology and Experimental Therapy, Wroclaw, Poland 2017

\begin{abstract}
The human gyrovirus-Apoptin (HGyv-Apoptin) is a protein that gained attention because it is selectively cytotoxic toward cancer cells. In this study, we have investigated the effect of HGyv-Apoptin on cell cycle progression of cancer cells. We also compared HGyv-Apoptin's action to its homologue chicken anemia virus Apoptin (CAVApoptin). We show that HGyv-Apoptin induces G2/M arrest in cancer cells. This is at least in part due to the fact that HGyv-Apoptin induces an abnormal spindle formation in mitotic cells that do not progress properly throughout the cell cycle. HGyv-Apoptin most likely inhibits APC function leading to a sustained cyclin-B1-expression. These results indicate that HGyv-Apoptin has a similar mechanism of action as its homolog CAV-Apoptin and further supports its cancer therapeutic potential.
\end{abstract}

Wiem Chaabane and Marek J. Łos share senior authorship.

Wiem Chaabane

wiemchaabane27@hotmail.fr

$\triangle$ Marek J. Łos

bioappl@gmail.com

1 Department of Biology, Faculty of Sciences, Tunis University, 2092 Tunis, Tunisia

2 Department of Human Anatomy and Cell Science, Faculty of Health Sciences, College of Medicine, University of Manitoba, Winnipeg, MB, Canada

3 Health Policy Research Center, Shiraz University of Medical Sciences, Shiraz, Iran

4 Faculty of Physiotherapy, The Jerzy Kukuczka Academy of Physical Education in Katowice, Katowice, Poland

5 ENT Department, School of Medicine, Medical University of Silesia in Katowice, Katowice, Poland
Keywords Apoptin $\cdot$ PKTCs $\cdot$ Cell cycle $\cdot$ APC $\cdot$ Cyclin B1

$\begin{array}{ll}\text { Abbreviations } & \\ \text { APC } & \text { Anaphase promoting complex } \\ \text { APC/C } & \text { Anaphase promoting complex/cyclosome } \\ \text { CAV } & \text { Apoptin, chicken anemia virus apoptin } \\ \text { CDK } & \text { Cyclin-dependent kinase } \\ \text { GFP } & \text { Green-fluorescent protein } \\ \text { Gyv-Apoptin } & \text { Human gyrovirus-Apoptin } \\ \text { PKTCs } & \text { Proteins killing tumor cells } \\ \text { PML } & \text { Promyelocytic leukemia }\end{array}$

\section{Introduction}

Apoptosis is a form of cell death, which is essential for normal development and for disease control via eliminating the defective cells (Los et al. 1999; Teodoro and Branton 1997). While apoptosis is highly regulated in normal tissue, most of the cancerous tissues show a deregulated apoptotic process due to the mutation of genes indispensable for apoptosis (Pettigrew and Cotter 2009), or genomic stability (Wang et al. 1997; Wiechec 2011). This enables the replication and proliferation of defective cells. While current/ old therapies rely on the targeting of one apoptotic abnormality and lack specificity, some of the newly emerged therapies aim the selective targeting of cancer tissues by the so-called PKTCs (proteins killing tumor cells) which are a family of proteins with cancer selective toxicity (Bruno et al. 2009; Rollano Penaloza et al. 2014).

Most of the PKTCs especially the viral PKTCs play a major role in cell cycle progression. The cell cycle progression is orchestrated by checkpoints at two major phases, the S-phase during which the DNA duplication occurs, and the 
M-phase during which the distribution of the genetic material into the two daughter cells takes place (Malumbres and Barbacid 2009). S-phase is preceded by a gap (G1), during which the cell prepares for DNA synthesis. The G1-checkpoint makes sure the internal and external conditions are right for division; this includes the cell size, availability of nutrients, positive molecular signals and DNA integrity. The S-phase is followed by a gap (G2) during which the cell prepares for mitosis. Prior to mitosis, the G2-checkpoint insures that DNA is completely replicated, that replications errors have been repaired, and that the cell is large enough to divide. During mitosis, the spindle assembly check point (SAC) ensures the fidelity of chromosome segregation to prevent chromosome mis-segregation and aneuploidy (Malumbres and Barbacid 2009). SAC is activated during prometaphase to ensure the fidelity of DNA replication. Activated SAC proteins inhibit the ability of anaphase promoting complex/cyclosome (APC/C) cdc20 to ubiquitinate two substrates: securin and cyclin B (Hwang et al. 1998; Kim et al. 1998). Securin inhibits separase, the enzyme that cleaves the cohesion between the sister chromatids and is thus required for the progression of cell division to anaphase (Panigrahi et al. 2008; Peters 2006). While cyclin B forms a complex with cyclin-dependent kinase 1 (CDK1) whose deactivation is essential for mitotic progression and exit (Peters 2006).

The adenoviral protein E4orF4 induces G2/M arrest and apoptosis in cancer cells in a p53-independent manner (Kornitzer et al. 2001). In addition to E4orF4, CAVApoptin, another avian-derived viral PKTC, also induces G2/M cell cycle arrest and apoptosis in a p53-independent manner in human malignant cells, while having no deleterious effect on normal cells. CAV-Apoptin shows a differential behavior in transformed and in primary cells. In cancer cells, CAV-Apoptin is phosphorylated on Threonine 108 and accumulates in the nucleus while it remains in the cytoplasm of normal cells, where it is degraded by the proteasome. The C-terminal end of CAV-Apoptin contains a nuclear localization sequence (NLS) and a CRM1-binding nuclear export sequence that are crucial for the nuclear export of CAV-Apoptin in the cells (Fig. 1). The nuclear localization of CAV-Apoptin is required for induction of apoptosis, which suggests that the cellular target of CAV-Apoptin is/are nuclear protein(s). CAV-Apoptin was shown to interfere with APC1, a subunit of APC/C. This interaction only occurs in cancer cells but not in normal cells. Domains that overlap with Apoptin NLS mediate CAV-Apoptin binding to $\mathrm{APC} 1$. This interaction disrupts the $\mathrm{APC} / \mathrm{C}$, via the nuclear sequestration of APC1, and maintains an active SAC leading to G2/M cell cycle arrest and apoptosis in a p53-independent manner (Teodoro et al. 2004).

A homolog of chicken anemia virus Apoptin (CAVApoptin), human gyrovirus-Apoptin (HGyv-Apoptin) also induces apoptosis selectively in cancer cells and functions independently from p53 (Chaabane et al. 2014; Rollano Penaloza et al. 2014). Little is known about the molecular mechanism of action of HGyv-Apoptin. HGyvApoptin has the same subcellular distribution as the CAV-Apoptin; it localizes in the nuclei of cancer cells where it shows a granular distribution that later clusters to form aggregates while it remains in the cytoplasm of normal cells (Bullenkamp et al. 2012). Despite a low overall identity important regions such as the nuclear localization and export signals and phosphorylation sites are conserved between HGyV and CAV-Apoptin suggesting a similar mechanism of action (Sauvage et al. 2011) (Fig. 2).

The purpose of this study is to investigate whether HGyv-Apoptin has a similar effect on cell cycle progression as its homolog CAV-Apoptin and functions via targeting the cell cycle, thus inducing G2/M arrest in cancer cells independently from $\mathrm{p} 53$. To this end, we have investigated the effect of HGyv-Apoptin on cell cycle progression. We show that HGyv-Apoptin induces abnormal spindle and G2/M arrest in cancer cells. HGyv-Apoptin seems to interfere with APC/C function, as cells expressing HGyv-Apoptin maintain high levels of CyclinB1 in cancer cells but not in normal cells, thus preventing the completion of the cell cycle and the mitotic exit.
Fig. 1 Diagrammatic representation of CAV-Apoptin functional domains (the number of aminoacids at each structural motif is indicated). $P M L$ promyelocytic leukemia, NES nuclear export sequence, $N L S$ nuclear localization sequence, $L R S$ leucine-rich sequence

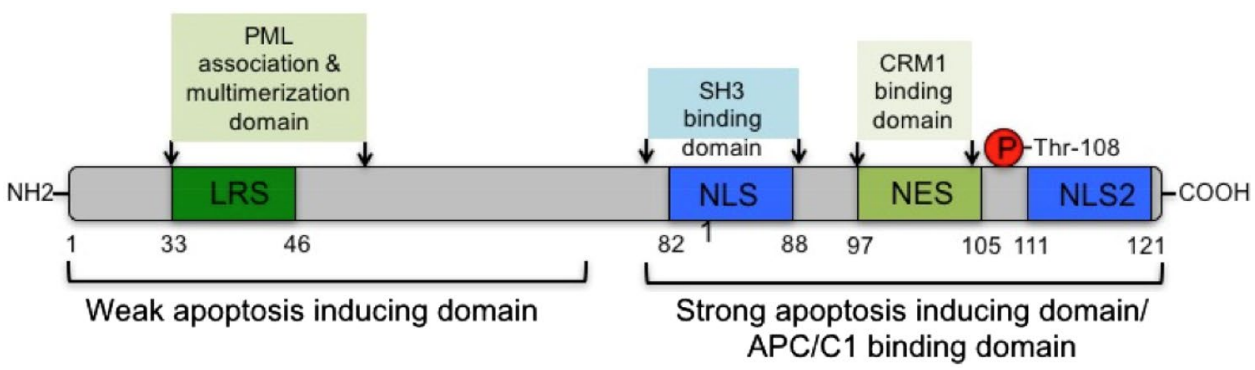




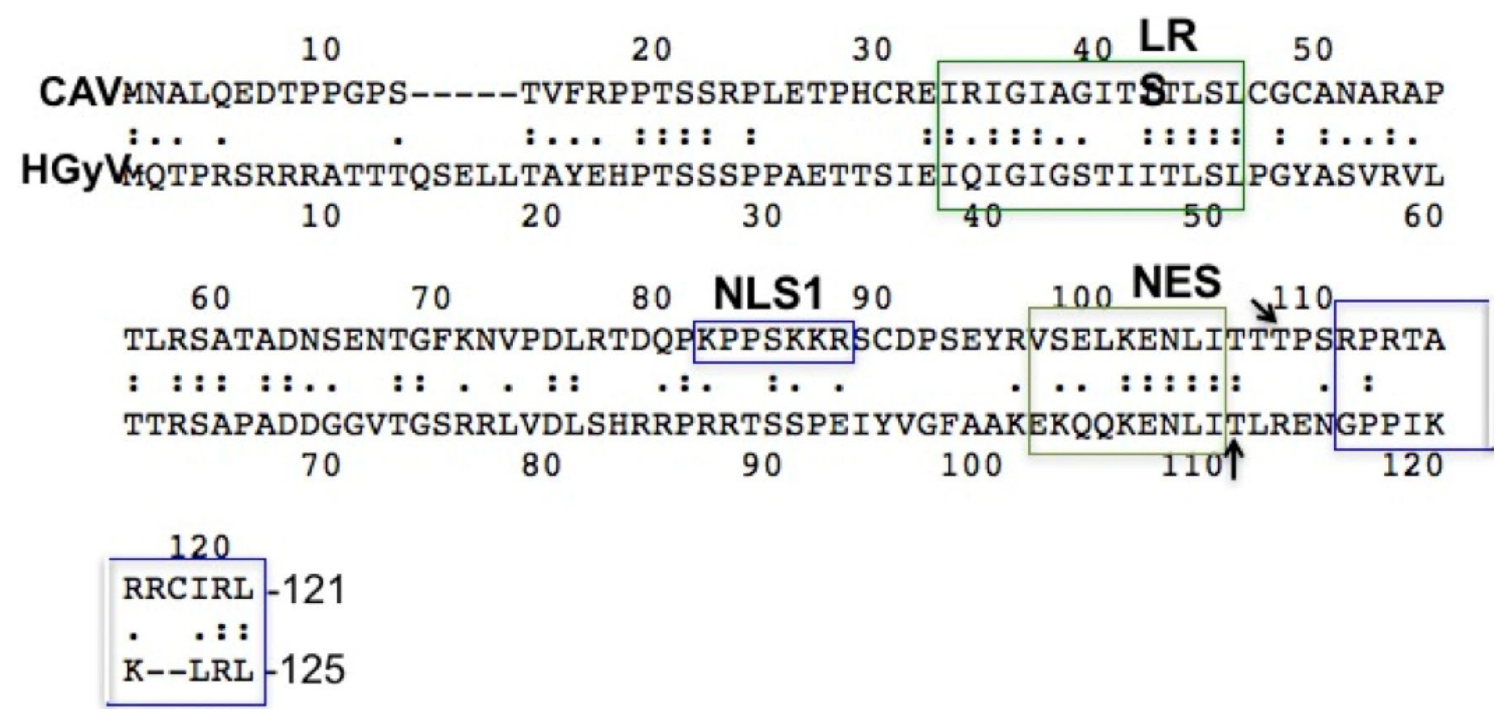

Fig. 2 Sequence alignment of CAV- and HgyV-Apoptin (http:// xylian.igh.cnrs.fr/bin/align-guess.cgi). Important functional domains, including LRS, NLS1/2 and nuclear export sequence (NES) are indi-

\section{Materials and Methods}

\section{Antibodies}

The following primary antibodies were used: mouse anti${ }^{\circledR}$ actin (Abcam, UK). $\alpha / \beta$-Tubulin Antibody (Cell signaling), Anti-cyclin B1 (Millipore, Germany), Anti-phosphoSer/Thr-Pro MPM-2 Antibody (Millipore, Germany). The following secondary antibodies were used: Infrared dye $800 \mathrm{cw}$ goat, anti-rabbit antibody, Infrared dye $680 \mathrm{cw}$ goat, anti-mouse antibody (Licor, USA), Rhodamine redX antirabbit antibody (life technologies), cy5 anti-murine antibody (Abcam, UK).

\section{Cell Culture and Reagents}

HCT116 (colon carcinoma) cells were grown in DMEM medium supplemented with $10 \%$ fetal calf serum (Hyclone), $100 \mu \mathrm{g} / \mathrm{ml}$ penicillin and $0.1 \mu \mathrm{g} / \mathrm{ml}$ streptomycin (Gibco BRL, New Zeland). Cells were cultured at $37^{\circ} \mathrm{C}$ with $5 \% \mathrm{CO}_{2}$ in a humidified incubator.

\section{Plasmids and Transient Transfections}

The following expression plasmids were used: GFP-HGyvapoptin (GFP-HGV-APT), GFP-CAV-apoptin (GFP-CAVAPT), and the negative control pEGFPC1. Cells were transfected with pEGFPC1 vector alone or with GFP-HGV-APT and GFP-CAV-APT using XtremeGENE HP DNA Transfection Reagent according to the manufacturer's instructions (Roche, Germany). cated by boxes and the predicted phosphorylation sites threonine 108 or threonine 111 are indicated by black arrows

\section{Cell Extracts and Immunoblotting}

The level of protein expression of cyclin B1 was detected by immunoblotting. Briefly, $2 \times 105$ cells were transfected with pEGFPC1 vector alone or with GFP-HGyv-apoptin. The expression of GFP and GFP-HGV-APT was confirmed by fluorescence microscopy. At the indicated time periods, whole cell lysates were prepared using RIPA Buffer and according to the manufacturer's instructions (Thermoscientific). Proteins $(30 \mu \mathrm{g})$ were separated by denaturing SDS-PAGE with $30 \% \mathrm{~T}$ acrylamide-Bisacrylamide and then transferred onto a PVDF membrane. The membranes were blocked in 5\% non-fat dried milk in TBS $0.1 \%$ tween and then incubated overnight with the following primary antibody rabbit anti-cyclin B1 (Millipore, Germany), and

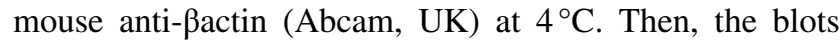
were incubated with the corresponding InfraRed dye secondary antibodies (LICOR). Visualization was carried out by a LICOR membrane scanner.

\section{Immunocytochemistry and Confocal Imaging}

Cells were grown overnight on coverslips and then transfected either with GFP-HGV-APT, GFP-CAV-APT or pEGFPC1. At the indicated time points, cells were washed with PBS and then fixed in $4 \%$ paraformaldehyde for 20 min (Cao et al. 2016; Heilman et al. 2006; Teodoro et al. 2004). Cells were then permeabilized with $0.1 \%$ Triton X-100 and blocked in $0.1 \%$ BSA. Cells were incubated with $\alpha / \beta$-Tubulin antibody overnight at $4{ }^{\circ} \mathrm{C}$, followed by three wash steps with PBS and $1 \mathrm{~h}$ incubation with the 
corresponding Rhodamine redX secondary antibody. The nuclei were visualized by DAPI staining. The fluorescent images were then observed and analyzed with a Zeiss LSM 510 inverted laser-scanning confocal fluorescence microscope using 40X oil planochromat objective (Carl Zeiss, Thornwood, NY).

\section{Cell Cycle Analysis}

Cells were washed in PBS, fixed in 70\% ethanol and stored at $4{ }^{\circ} \mathrm{C}$ for $1 \mathrm{~h}$. Cells were then resuspended in $1 \%$ FCS in PBS and stained with $50 \mu \mathrm{l}$ MPM-2 antibody overnight at $4{ }^{\circ} \mathrm{C}$. Next morning, cells were washed thrice in PBS. And then stained with anti-cy5 secondary antibody for $1 \mathrm{~h}$ at RT, cells were then washed thrice in PBS and resuspended in PBS containing $40 \mu \mathrm{g} / \mathrm{ml}$ of propidium iodide (PI) for 30 min followed by Analysis by flowcytometry using FL1 for FITC, FL4 for cy5 and FL3 for PI.

\section{Results}

\section{HGyv-Apoptin Induces G2/M Arrest Selectively in Cancer Cells}

We first checked the effect of HGyv-Apoptin on cell cycle progression. For this purpose, HCT116 cycling cells were transfected with GFP-HGyv-apoptin; pEGFPC1 was used as a negative control while GFP-CAV-apoptin was used as a positive control. The cells were stained with MPM2 and PI and analyzed by flow cytometry to determine the percentage of cells in each phase of the cell cycle. PI staining determines the DNA content while the cells positive for MPM2 are the mitotic cells. As by entry to mitosis many proteins are phosphorylated either directly or indirectly by M-phase-promoting factor, MPM2 stains phosphorylated M-phase proteins, giving an indication about the number of cells on the M-phase of the cell cycle.

As shown in Fig. 3, HCT116 cells that either express HGyv-Apoptin or CAV-Apoptin show a G2/M arrest as compared to the cells that express the control vector. G2/M arrest occurs prior to apoptosis as upon HGyv-Apoptin expression; cells arrest at the G2 phase with a sub-G1 degraded DNA content cells undergoing apoptosis starting to accumulate.

\section{HGyv-Apoptin Induces an Abnormal Spindle Formation}

To further investigate the effect of HGyv-Apoptin on cell cycle, we have checked the effect of HGyv-Apoptin expression on HCT116 mitotic cells. Recent studies have shown that CAV-Apoptin induces abnormal spindle formation in cancer cells leading to mitotic catastrophe (Lanz et al. 2013). To check whether HGyv-Apoptin has a similar effect, cells were stained with endogenous Tubulin to check bipolar spindles formation. Cells that express HGyv-Apoptin show an abnormal spindle as compared to the control cells. We have scored the number of cells expressing either of the vectors and showing an abnormal spindle. The results prove that HGyv-Apoptin induces an abnormal spindle formation in mitotic cells. HGyv-Apoptin mitotic expressing cells with abnormal spindle were greatly enlarged as compared to the control cells (Fig. 4). These data further support that HGyv-Apoptin has a similar mechanism of action as its homologue CAV-Apoptin.

\section{HGyv-Apoptin Effects on Cell Cycle Regulators}

To further understand HGyv-Apoptin mechanism of action, we have studied its effects on cell cycle regulators. The APC/C ubiquitination complex plays a major role in mitosis, with its cdc20 subunit critical for meta- to anaphase transition, and its CDK/Cyclin subunit regulating the mitotic exit. The ubiquitination of cyclin B allows for inactivation of CDK1/Cyclin B complex and the mitotic exit while CDK1/cyclin B1 complex prevents cell cycle exit (Malumbres and Barbacid 2005).

We thus evaluated the level of cyclin B1 expression in HCT116 cells that express either HGyv-Apoptin or CAVApoptin as compared to the cells that express the control empty GFP vector. HGyv-Apoptin as well as CAV-Apoptin expressing cells show a sustained high level of cyclin B1, as compared to the positive control HCT116 cells treated with the cell cycle arrest inducer Nocodazole, or the negative control HCT116 cells that were transfected with the control vector. In addition, normal primary human fibroblasts expressing HGyv-Apoptin show low level of cyclin B1 consistent with a normal progression of the cells through the cell cycle. These data suggest that HGyv-Apoptin interferes with the cell cycle most likely via inhibiting the APC function, thus blocking the exit from the cell cycle selectively in cancer cells while having no effect on cell cycle progression in normal cells (Fig. 5).

\section{Discussion}

Two viral PKTCs E4orF4 and CAV-Apoptin kill cancer cells by interference with the cell cycle, and by induction of G2/M arrest and apoptosis (Kornitzer et al. 2001; Lanz et al. 2013; Maddika et al. 2009). Earlier research from our group shows that CAV-apoptin presence in the cell leads to the activation of CDK2, and that the activated CDK2 is responsible for the phosphorylation of CAVApoptin at critical for nuclear retention, Thr-108 (Maddika 

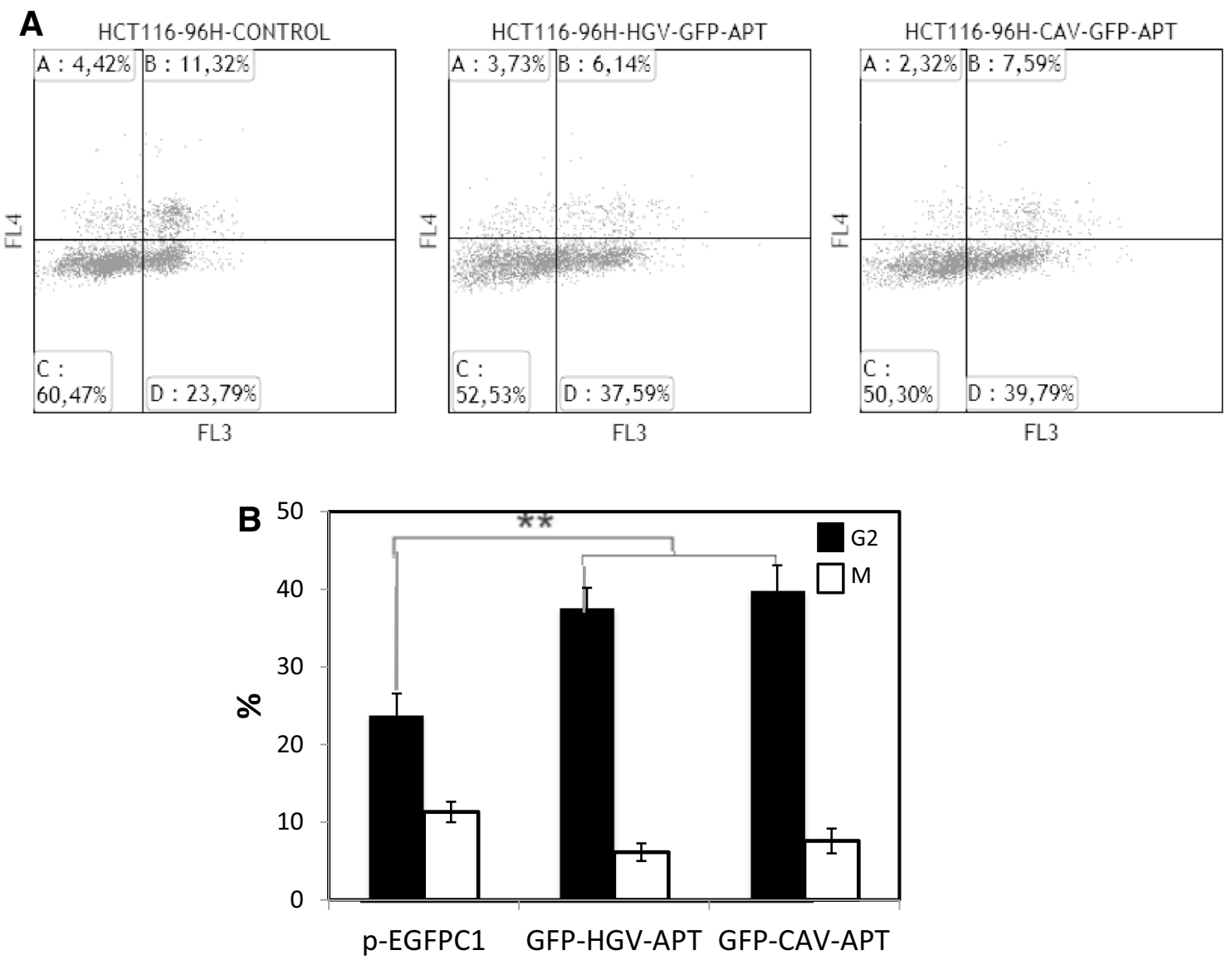

Fig. 3 HGyv-Apoptin induces G2/M arrest. a Cell cycle analysis using PI and MPM2 staining (indicator of phosphorylated M-phase proteins and the percentage of cells on the M-phase of the cell cycle). At the indicated time points, post-transfection, cells were stained overnight with anti-MPM-2 antibody, then washed three times the next morning, and stained with the anti-cy5 secondary antibody, followed by propidium iodide staining. While the cells that express the control vector progress normally through $>h$ out the cell cycle, cells that either express HGyv-Apoptin or CAV-Apoptin show a G2/M arrest. G2/M arrest occurs prior to apoptosis as upon HGyvApoptin or CAV-Apoptin expression; cells arrest at the G2 phase with an accumulation of sub-G1 degraded DNA content cells undergoing apoptosis. Green (FL-1, GFP-positive) cells were gated and analyzed

et al. 2009). CAV-Apoptin also causes nuclear transfer of Akt kinase that then phosphorylates in the nucleus several proteins contributing to CAV-Apoptin's toxicity (Jain et al. 2016; Maddika et al. 2007). Our presented data here indicate that HGyv-Apoptin also follows the same toxicity mechanism to kill tumor cells (Fig. 6). All the viral PKTCs interfere with the APC/C function suggesting a common mechanism for the detection and the elimination of tumor cells undoubtedly associated with viral replication.

To further understand viral PKTCs behavior in tumor cells and their selective toxicity which is highly associated with their nuclear localization and accumulation, it would be important to understand the significance of the among the whole population of cells. FL3 corresponds to linear propidium iodide fluorescence measurement and FL4 corresponds to cyc 5 integrated fluorescence measurements. b Quantification of the percentage of cells at the G2 (black bars) or M phase (white bars) of the cell cycle, cells that either express HGyv-Apoptin or CAV- Apoptin show an arrest at the $\mathrm{G} 2$ phase of the cell cycle as compared to the cells that express the control vector. The mean of G2 arrested cells was calculated from three independent experiments. Both HGyvApoptin and CAV-Apoptin show significantly more G2-arrested cells than the control group $(P<0.01)(P=6.0300044652859 \mathrm{E}-11$ for HGyv-Apoptin cells and 3.6211569068185E-11 for CAV-Apoptin cells)

nuclear accumulation of HGyv-Apoptin in cancer cells, and to identify its nuclear interacting partners. Studies have shown that CAV-Apoptin shuttles in both tumor and normal cells but only accumulates in the nuclei of tumor cells, we tempt to speculate that apoptins may sequestrate components essential for the cell cycle progression like APC1. For example, APC1 may be sequestrated in promyelocytic leukemia bodies by CAV-Apoptin (Heilman et al. 2006). It would be interesting to check if HGyv-Apoptin shows the same cellular behavior, to identify other nuclear interacting partners and to understand to which extent this shuttling is essential for its apoptotic activity. In the proposed model, the inhibition of the nuclear export of HGyv-Apoptin 
Fig. 4 HGyv-Apoptin induces an abnormal spindle. a Spindle formation in HGyv-Apoptin expressing HCT116 cells. Cells were fixed $48 \mathrm{~h}$ posttransfection and stained with DAPI for the detection of nuclear morphology and with anti-tubulin antibody for the detection of spindle formation. b Quantification of the percentage of cells with abnormal spindle and either expressing the control vector or HGyvApoptin. Per experiment, 100 mitotic cells that were positive for GFP-HGyv-Apoptin and pEGFC1 were scored. HGyvApoptin-positive mitotic cells contained significantly more abnormal spindles $(P<0.01)$ than the control vector $(P=2.2006711030126 \mathrm{E}-11)$
A

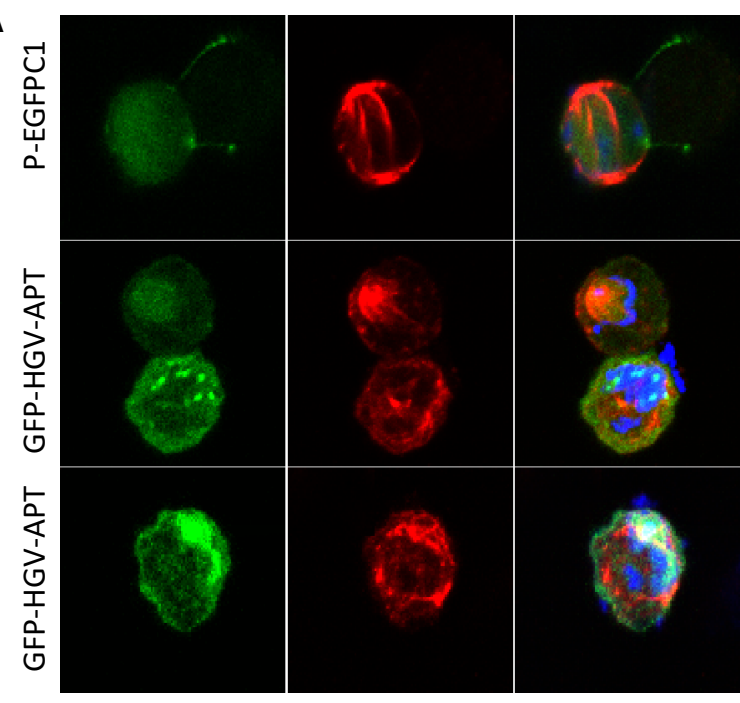

B

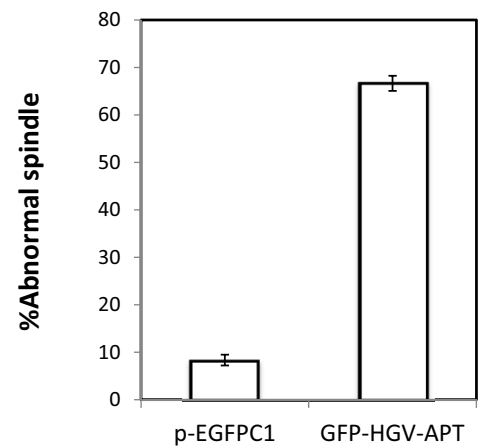

A
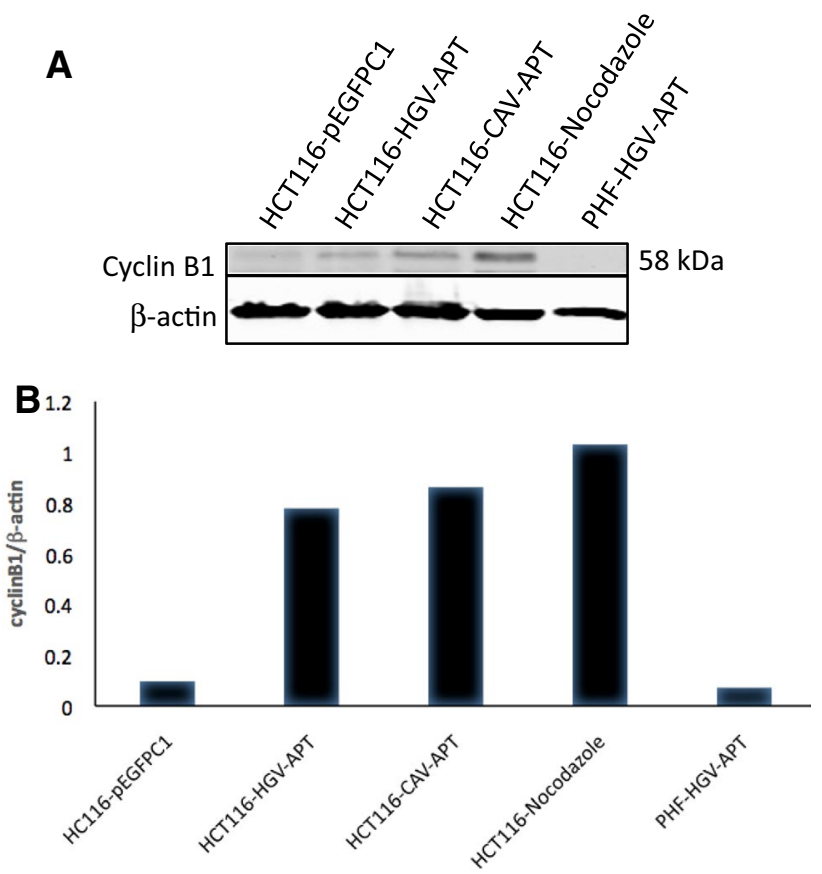

Fig. 5 HGyv-Apoptin effects on cell cycle regulators. a HGyv-Apoptin expression results in sustained levels of Cyclin B1 expression, showing that HGyv-Apoptin interferes with the APC/C ubiquitin function. The decline of cyclin B through degradation by APC/C is required for the completion and the exit from the cell cycle. b Densitometric representation of cyclinB1/beta-actin ratio from "A" should abolish its toxicity. In this case, a decreased apoptosis and a decreased nuclear level of HGyv-Apoptin should be observed. To test the proposed model of HGyv-Apoptin action, one could employ HGyv-Apoptin mutants lacking ability to enter the nucleus, either by mutating critical elements of the bipartial nuclear localization signal, or by mutating the critical Threonine close to the C-terminus of the protein.

Viruses may not only be the source of proteins with selective anticancer properties, but also may contribute to carcinogenesis, like for example human papillomaviruses (cervical and oral cancer) (Liu et al. 2016; Zaravinos 2014), human T-cell leukemia virus (hairly cell leukemia) (Chlichlia et al. 2002; Los et al. 1998) etc. Like in some other diseases (Likus et al. 2016a), it is however sometimes difficult to clearly associate the given pathogen with cancer etiology. Many viruses preferentially or exclusively replicate in dividing cells; hence, their components are potentially becoming an important source/inspiration in the search of drugs that preferentially target cancer stem cells (AkbariBirgani et al. 2016; Farahani et al. 2014; Wasik et al. 2014). Beside viral methods, some clinically tested as well as new drugs with preferential toxicity towards cancer stem cells are increasingly becoming available (Jangamreddy et al. 2013; Likus et al. 2016b; Moosavi et al. 2016). Such tasks are facilitated by recent progress in methodologies that allows for better detection and monitoring of cancer stem 
Fig. 6 Proposed model for HGyv-Apoptin mechanism of action. In cancer cells, nuclear HGyv-apoptin induces abnormal spindle formation and serves most likely to the nuclear sequestration of components essential for the cell cycle progression like APC1. This leads to a weakened SAC and sustained high levels of cyclin B1 leading to G2/M arrest and apoptosis

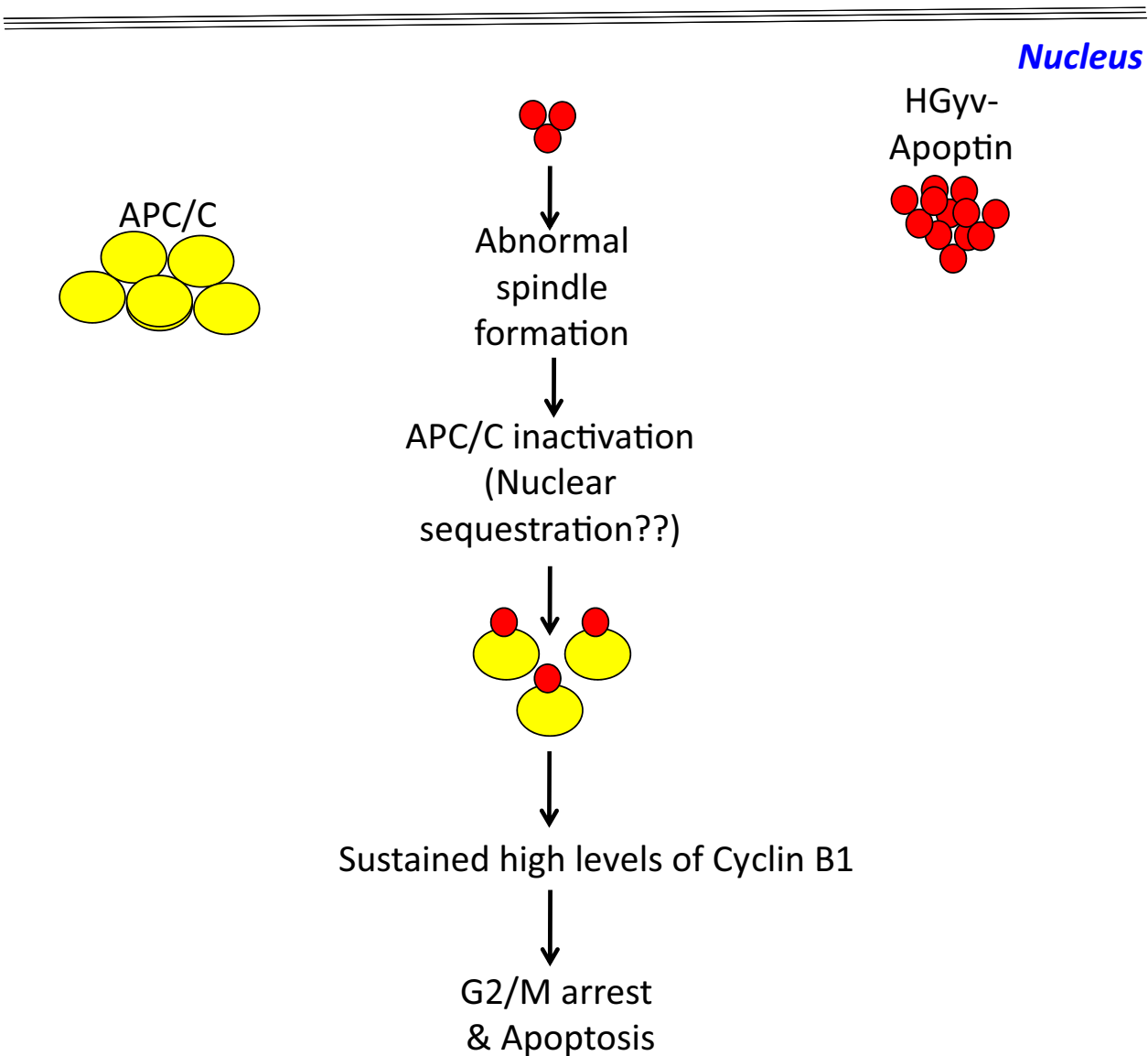

cells (Cieslar-Pobuda et al. 2015, 2016; Likus et al. 2016c). While it is unlikely that a universal cancer cure will be found, rapid development will certainly lead to efficient anticancer strategies targeting groups of cancers.

Acknowledgements SG acknowledges Manitoba Medical Service Foundation, and University of Manitoba Research Grant Program. MJŁ kindly acknowledges the support from NCN Grant \#: 2016/21/B/ NZ1/02812.

\section{References}

Akbari-Birgani S, Paranjothy T, Zuse A et al (2016) Cancer stem cells, cancer-initiating cells and methods for their detection. Drug Discov Today 21:836-842

Bruno P, Brinkmann CR, Boulanger MC et al (2009) Family at last: highlights of the first international meeting on proteins killing tumour cells. Cell Death Differ 16:184-186

Bullenkamp J, Cole D, Malik F et al (2012) Human Gyrovirus Apoptin shows a similar subcellular distribution pattern and apoptosis induction as the chicken anaemia virus derived VP3/Apoptin. Cell Death Dis 3:e296

Cao HH, Chu JH, Kwan HY et al (2016) Inhibition of the STAT3 signaling pathway contributes to apigenin-mediated anti-metastatic effect in melanoma. Sci Rep 6:21731
Chaabane W, Cieslar-Pobuda A, El-Gazzah M et al (2014) Humangyrovirus-apoptin triggers mitochondrial death pathway-Nur77 is required for apoptosis triggering. Neoplasia 16:679-693

Chlichlia K, Los M, Schulze-Osthoff K et al (2002) Redox events in HTLV-1 Tax-induced apoptotic T-cell death. Antioxid Redox Signal 4:471-477

Cieslar-Pobuda A, Jain MV, Kratz G et al (2015) The expression pattern of PFKFB3 enzyme distinguishes between induced-pluripotent stem cells and cancer stem cells. Oncotarget 6:29753-29770

Cieslar-Pobuda A, Rafat M, Knoflach V et al (2016) Human induced pluripotent stem cell differentiation and direct transdifferentiation into corneal epithelial-like cells. Oncotarget 7:42314-42329

Farahani E, Patra HK, Jangamreddy JR et al (2014) Cell adhesion molecules and their relation to (cancer) cell stemness. Carcinogenesis 35:747-759

Heilman DW, Teodoro JG, Green MR (2006) Apoptin nucleocytoplasmic shuttling is required for cell type-specific localization, apoptosis, and recruitment of the anaphase-promoting complex/cyclosome to PML bodies. J Virol 80:7535-7545

Hwang LH, Lau LF, Smith DL et al (1998) Budding yeast Cdc20: a target of the spindle checkpoint. Science 279:1041-1044

Jain MV, Shareef A, Likus W et al (2016) Inhibition of miR301 enhances Akt-mediated cell proliferation by accumulation of PTEN in nucleus and its effects on cell-cycle regulatory proteins. Oncotarget 7:20953-20965

Jangamreddy JR, Ghavami S, Grabarek J et al (2013) Salinomycin induces activation of autophagy, mitophagy and affects 
mitochondrial polarity: differences between primary and cancer cells. Biochim Biophys Acta 1833:2057-2069

Kim SH, Lin DP, Matsumoto S et al (1998) Fission yeast Slp1: an effector of the Mad2-dependent spindle checkpoint. Science 279:1045-1047

Kornitzer D, Sharf R, Kleinberger T (2001) Adenovirus E4orf4 protein induces PP2A-dependent growth arrest in Saccharomyces cerevisiae and interacts with the anaphase-promoting complex/cyclosome. J Cell Biol 154:331-344

Lanz HL, Zimmerman RM, Brouwer J et al (2013) Mitotic catastrophe triggered in human cancer cells by the viral protein apoptin. Cell Death Dis 4:e487

Likus W, Siemianowicz K, Markowski J et al (2016a) Bacterial infections and osteoclastogenesis regulators in men and women with cholesteatoma. Arch Immunol Ther Exp 64:241-247

Likus W, Siemianowicz K, Bienk K et al (2016b) Could drugs inhibiting the mevalonate pathway also target cancer stem cells? Drug Resist Updates 25:13-25

Likus W, Gruszczynska K, Markowski J et al (2016c) Correlations between selected parameters of nasal cavity in neonates and young infants—computed tomography study. Folia Morphol 75:334-340

Liu X, Gao XL, Liang XH et al (2016) The etiologic spectrum of head and neck squamous cell carcinoma in young patients. Oncotarget 7:66226-66238

Los M, Khazaie K, Schulze-Osthoff K et al (1998) Human T cell leukemia virus-I (HTLV-I) Tax-mediated apoptosis in activated T cells requires an enhanced intracellular prooxidant state. J Immunol 161:3050-3055

Los M, Wesselborg S, Schulze-Osthoff K (1999) The role of caspases in development, immunity, and apoptotic signal transduction: lessons from knockout mice. Immunity 10:629-639

Maddika S, Bay GH, Kroczak TJ et al (2007) Akt is transferred to the nucleus of cells treated with apoptin, and it participates in apoptin-induced cell death. Cell Prolif 40:835-848

Maddika S, Panigrahi S, Wiechec E et al (2009) Unscheduled Akttriggered activation of cyclin-dependent kinase 2 as a key effector mechanism of apoptin's anticancer toxicity. Mol Cell Biol 29:1235-1248
Malumbres M, Barbacid M (2005) Mammalian cyclin-dependent kinases. Trends Biochem Sci 30:630-641

Malumbres M, Barbacid M (2009) Cell cycle, CDKs and cancer: a changing paradigm. Nat Rev Cancer 9:153-166

Moosavi MA, Sharifi M, Ghafary SM et al (2016) Photodynamic $\mathrm{N}-\mathrm{TiO} 2$ nanoparticle treatment induces controlled ROS-mediated autophagy and terminal differentiation of leukemia cells. Sci Rep 6:34413

Panigrahi S, Klonisch T, Los M (2008) The art of killing: double stroke with apoptin and survivin as a novel approach in cancer therapy. Cancer Biol Ther 7:1061-1062

Peters JM (2006) The anaphase promoting complex/cyclosome: a machine designed to destroy. Nat Rev Mol Cell Biol 7:644-656

Pettigrew CA, Cotter TG (2009) Deregulation of cell death (apoptosis): implications for tumor development. Discov Med 8:61-63

Rollano Penaloza OM, Lewandowska M, Stetefeld J et al (2014) Apoptins: selective anticancer agents. Trends Mol Med 20:519-528

Sauvage V, Cheval J, Foulongne V et al (2011) Identification of the first human gyrovirus, a virus related to chicken anemia virus. J Virol 85:7948-7950

Teodoro JG, Branton PE (1997) Regulation of apoptosis by viral gene products. J Virol 71:739-746

Teodoro JG, Heilman DW, Parker AE et al (2004) The viral protein Apoptin associates with the anaphase-promoting complex to induce $\mathrm{G} 2 / \mathrm{M}$ arrest and apoptosis in the absence of $\mathrm{p} 53$. Genes Dev 18:1952-1957

Wang ZQ, Stingl L, Morrison C et al (1997) PARP is important for genomic stability but dispensable in apoptosis. Genes Dev 11:2347-2358

Wasik AM, Grabarek J, Pantovic A et al (2014) Reprogramming and carcinogenesis-parallels and distinctions. Int Rev Cell Mol Biol 308:167-203

Wiechec E (2011) Implications of genomic instability in the diagnosis and treatment of breast cancer. Expert Rev Mol Diagn $11: 445-453$

Zaravinos A (2014) An updated overview of HPV-associated head and neck carcinomas. Oncotarget 5:3956-3969 